\title{
Co-delivery of Doxorubicin and PSC 833 (Valspodar) by Stealth Nanoliposomes for Efficient Overcoming of Multidrug Resistance
}

\author{
Elmira Bajelan ${ }^{1}$, Azadeh Haeri $^{1}$, Amir Masoud Vali ${ }^{2,}$ Seyed Nasser Ostad ${ }^{3}$, Simin Dadashzadeh $^{1,4, *}$ \\ ${ }^{1}$ Department of Pharmaceutics, School of Pharmacy, Shahid Beheshti University of Medical Sciences, Tehran, Iran. \\ ${ }^{2}$ Department of Pharmaceutics, School of Pharmacy, Zanjan University of Medical Sciences, Zanjan, Iran. \\ ${ }^{3}$ Department of Toxicology and Pharmacology and Nanotechnology Research Center, Faculty of Pharmacy, Tehran \\ University of Medical Sciences, Tehran, 14155/6451, Iran. \\ ${ }^{4}$ Pharmaceutical Sciences Research Center, Shahid Beheshti University of Medical Sciences, Tehran, Iran.
}

Received, April 21, 2012; Revised, July 31, 2012; Accepted, October 5, 2012; Published, October 7, 2012.

\begin{abstract}
Purpose. This study was aimed at developing co-encapsulated stealth nanoliposomes containing PSC 833, an efficient MDR modulator, and doxorubicin (DOX) in order to increase the effectiveness and decrease adverse effects of the anticancer drug. Methods. In attempt to increase the encapsulation efficiency of drugs, different methods for liposome preparation were tested and the effect of different parameters such as drug to lipid molar ratio, cholesterol mole percent and lipid compositions, were investigated. The final product with a lipid composition of EPC:DSPE-PEG2000:Chol (60:5:30\%mol) was prepared by thin layer film hydration method. After preparation of empty liposomes, DOX and PSC 833 were loaded using ammonium sulfate gradient and remote film loading methods, respectively. Physical characteristics of optimized liposomes (DOX/PSC-L) such as particle size, zeta potential, encapsulation efficiency, in-vitro drugs release and stability were evaluated. Furthermore, in vitro cytotoxicity study of various liposomal formulations as well as drugs, solutions against resistant human breast cancer cell line, T47D/TAMR-6, was evaluated using MTT assay. Results. The best formulation showed a narrow size distribution with average diameter of $91.3 \pm 0.2 \mathrm{~nm}$ with zeta potential of $-6 \pm 1.2$, the encapsulation efficiency for DOX and PSC 833 were more than $95 \%$ and $65.5 \%$, respectively. In DOX-resistant T47D/TAMR-6 cells, dual-agent stealth liposomes showed significantly greater cytotoxicity $(P<0.05)$ than free DOX and liposomal DOX plus free PSC 833 treatments. Conclusions. Co-encapsulation of DOX and PSC 833 presents a promising anticancer formulation, capable of effective reversal of drug resistance, and should be explored further in therapeutic studies with animal tumor xenograft models.
\end{abstract}

This article is open to POST-PUBLICATION REVIEW. Registered readers (see "For Readers") may comment by clicking on ABSTRACT on the issue's contents page.

\section{INTRODUCTION}

Chemotherapeutics are the mainstream strategy for the treatment of localized and metastasized cancers. However, the development of multidrug resistance (MDR) of cancer cells, as well as systemic toxic side effects resulting from unspecific localization of anticancer drugs to nontumor areas are major obstacles to the success of chemotherapy in many cancers $(1,2)$. MDR can be caused by drug efflux transporters which actively efflux out the drug, leading to reduced intracellular drug accumulation and decreased therapeutic efficacy $(1,3)$.

P-glycoprotein (P-gp), encoded by the MDR1 gene, is the most abundantly expressed drug efflux system in cell membranes, which actively extrude a diverse range of structurally and functionally unrelated chemotherapeutic drugs such as, anthracyclines, vinca alkaloids and taxanes against a concentration gradient from the cell $(4,5)$. Role of P-gp in clinical tumor resistance is supported by studies that demonstrate P-gp expression in more than $40 \%$ of breast cancer samples and its correlation with decreased treatment response $(6,7)$.

The role of P-gp in MDR has led to efforts to modulate P-gp activity. A number of compounds that possess P-gp inhibitory activities, which are sometimes referred as "chemosensitizers", have been identified or synthesized to address this issue (8). First-generation inhibitors are pharmacologically active compounds, which are in clinical use for other indications but have been shown to inhibit P-gp. These include calcium channel blockers such as verapamil and immunosuppressants like cyclosporin A.

Corresponding Author: S. Dadashzadeh, Professor, Department of Pharmaceutics, School of Pharmacy, Shahid Beheshti University of Medical Sciences, Tehran, Iran. Email address: Sdadashzadeh@sbmu.ac.ir (S. Dadashzadeh) 
However, significant cardiac toxicities, immunosuppressive and nephrotoxic effects, low potencies and poor specificity for the drug efflux transporters limit their clinical use $(9,10)$. PSC 833 (Valspodar) is an analogue of cyclosporin, which is more active in vitro and in vivo than cyclosporin A or other agents such as verapamil and quinidine in reversing P-gp mediated multidrug resistance. This agent lacks nephrotoxicity or immunosuppressive activity. The anticancer efficacy of the combination of PSC 833 and P-gp substrate anticancer drugs in preclinical studies yielded greater reduction of tumor size and longer life span than the individual anticancer compound in animals bearing MDR tumors $(11,12)$. Furthermore, the P-gp inhibiting activity of PSC- 833 has been demonstrated in clinical trials in combination with chemotherapeutic agents $(13,14)$. In spite of high potency and specificity of the newer agents such as PSC 833, a major confounding factor in their use is the fact that besides exerting an effect on Pgp function, they also have a profound effect on pharmacokinetics and cytotoxicity of the anticancer drugs concurrently administered (15). The pharmacokinetic interaction and increase in cytotoxicity of anticancer drugs by a MDR modulator is due to extensive biodistribution of the MDR modulators in normal organs, such as intestine, liver, kidneys, lung and brain, inhibiting P-gp function in these tissues and causing increased distribution or decreased excretion of the chemotherapeutics (16). Notably, another important concern is that the difference in physico-chemical properties of the anticancer drug with chemosensitizer may result in differences in the pharmacokinetics and accumulation of the two agents in tumor site. Therefore, concurrent administration of efflux inhibitor and anticancer drug cannot guarantee the co-action of intended drugs in the same cancer cells (17). A great deal of these shortcomings of chemosensitizers may be tackled by administering these agents using nanoparticulate delivery systems (18).
In particular, co-encapsulation of dual agents into a nanoparticle formulation would be more effective than concurrent application of single agent-containing nanoparticles, since any nanoparticle uptake by the cell would result in coexistence of the chemosensitizer and the anticancer drug in the microdomain of the cell and the exploitation of the synergy (17). Furthermore, simultaneous delivery of both drugs by a particle formulation allowed for ease of administration. It should be noted that encapsulation of anticancer drugs in nanocarriers in turn can reduce exposure of entrapped drugs to susceptible healthy tissues while preferentially accumulating in sites of tumor growth due to enhanced permeability and retention (EPR) effects associated with solid tumors $(19,20)$.

Over the past few decades, liposomes have received widespread attention as a carrier system for therapeutically active compounds, due to their unique characteristics such as capability to incorporate hydrophilic and hydrophobic drugs, good biocompatibility, low toxicity, lack of immune system activation, and targeted delivery of bioactive compounds to the site of action (21, 22). Recently, a novel liposomal formulation containing co-loaded doxorubicin (DOX) and verapamil was synthesized and shown to overcome MDR $(23,24)$. The chemosensitizer verapamil chosen in this study, however, was non-specific for P-gp and would cause side effect. Therefore, the present work was undertaken to develop a new liposomal formulation loaded with both DOX and the much more potent and specific P-gp modulator, PSC 833. In the present study, the preparation and characterization of PSC 833 loaded liposomes (PSC-L) and combined liposomal formulation (DOX/PSC-L) were investigated. In addition, the cytotoxicity of various formulations was verified on human breast cancer resistant cell line. This approach ameliorates extensive biodistribution of both drugs and allows for enhanced antitumor efficacy to tumor cells.

\section{ABBREVIATIONS}

\%DR: percent drug released; $\mathbf{\%} \mathbf{D R}_{\mathbf{2 4 h}}$ : percent drug released after $24 \mathrm{~h} ; \mathbf{\%} \mathbf{O R}_{\mathbf{4 8 h}}$ : percent drug released after 48 h; Chol: cholesterol; DOX: doxorubicin; DOX-L: doxorubicin entrapped liposome; DOX/PSC-L: doxorubicin and PSC 833 entrapped liposome; DPPC: dipalmitoylphosphatidylcholine; DSPC: distearoylphosphatidylcholine; DSPE-PEG: distearoylphosphatidylethanolamine-poly(ethyleneglycol)2000; EE: entrapment efficiency; EL: empty liposomes; EPC: egg phosphatidylcholine; FBS: fetal bovine serum; MDR: multidrug resistance; MTT: 3-(4, 5-dimethyltiazol-2-ly)-2, 5-diphenyltetrazolium bromide; PC: phosphatidylcholine; P-gp: P-glycoprotein; PSC-L: PSC 833 entrapped liposome; $\mathbf{T}_{\mathbf{m}}$ : transition temperature. 


\section{MATERIALS AND METHODS}

\begin{abstract}
Materials
PSC 833 was kindly provided by Novartis Pharmaceuticals (Switzerland). Purified egg phosphatidylcholine (EPC), dipalmitoylphosphatidylcholine (DPPC), distearoylphosphatidylcholine (DSPC) and distearoylphosphatidylethanolamine - poly (ethyleneglycol) 2000 (DSPE-PEG2000) were obtained from Lipoid GmbH (Switzerland). Doxorubicin hydrochloride (DOX), tamoxifen, cholesterol (Chol, purity > 99\%), 3-(4, 5-dimethyltiazol-2ly)-2, 5-diphenyltetrazolium bromide (MTT) and ammonium sulfate were purchased from Sigma Chemical Company (St. Louis, MO, USA) and RPMI1640 and fetal bovine serum (FBS) from Gibco BRL (UK). Chloroform, methanol, HPLCgrade acetonitrile, Tween 80 , Triton X-100 and sodium chloride were supplied by Merck (Darmstadt, Germany). Cellulose dialysis tubing (molecular weight cutoff $12000 \mathrm{Da}$ ) was from BioGene (USA). Other supplements for cell culture media were obtained from Gibco Invitrogen (UK).
\end{abstract}

\section{Cell lines and culture conditions}

The human breast cancer cell line, T47D, (ATCC, HTB-133) was obtained from Pasteur Institute Cell Bank of Iran (Tehran, Iran). A subpopulation of T47D (T47D/TAMR-6) which shows resistance to tamoxifen and DOX and overexpresses MDR1 (25) was kindly provided by Prof. Azizi in Molecular Research Laboratory, Faculty of Pharmacy, Tehran University of Medical Sciences (Tehran, Iran). T47D/TAMR-6 was significantly less sensitive to the cytotoxic effects of DOX when compared to parental cells (see results). The cell cultured medium was RPMI 1640 which was supplemented with $10 \%$ heatinactivated FBS and $1 \%$ antibiotics (penicillin/streptomycin; $100 \mathrm{U} / \mathrm{ml}$, Gibco). T47D/TAMR-6 was cultured in the medium containing $10^{-6} \mathrm{M}$ tamoxifen for maintaining MDR phenotype. Cells were maintained at $37^{\circ} \mathrm{C}$ and $5 \% \mathrm{CO}_{2}$ in a humidified incubator.

\section{Liposome preparation}

For liposmal encapsulation of DOX (DOX-L) a transmembrane $\mathrm{pH}$ gradient method was carried out (26). PSC 833 loaded liposomes (PSC-L) were prepared by the remote film loading method, which was reported by Sadzuka et al. (27) as a novel method for liposome preparation and optimized in our previous study for sirolimus liposomalization (28). This method involves production of drug film and the subsequent addition to empty liposomes (ELs).

\section{Preparation of Empty liposomes}

ELs were prepared by the lipid film hydration method (29). Briefly, the lipid mixture of the desired molar composition was dissolved in chloroform and dried under reduced pressure in a rotary evaporator $(90 \mathrm{rpm})$ at $65^{\circ} \mathrm{C}$ to form a thin lipid film. Evaporation was continued for $2 \mathrm{~h}$ after the dry residue appeared, to completely remove all traces of the solvent. The film was then hydrated with ammonium sulfate $(300 \mathrm{mM})$ at $65^{\circ} \mathrm{C}$ for $1 \mathrm{~h}$. The obtained multivesicular suspensions were extruded (Northern Lipids, Vancouver, BC, Canada) five times through each of 200 and $100 \mathrm{~nm}$ pore size (Nucleopore polycarbonate membranes, Whatman, UK) to produce samples with a narrow size distribution. The extrusion was carried out at $65^{\circ} \mathrm{C}$ to maintain vesicles above phase transition temperature. Phospholipid content of the liposomes was assayed using the method established by Stewart (30).

\section{Liposomal encapsulation of doxorubicin}

DOX was remote-loaded using an ammonium sulfate gradient as previously described (26). The resulting ELs were dialyzed for $12 \mathrm{~h}$ at room temperature against $150 \mathrm{mM} \mathrm{NaCl}$ to exchange the external buffer and establish a $\mathrm{pH}$ gradient across the liposome membrane. DOX in normal saline was then mixed and incubated with ELs at $60^{\circ} \mathrm{C}$ for $1 \mathrm{~h}$. Small amounts of residual nonencapsulated DOX were removed by dialysis for $12 \mathrm{~h}$ at $4^{\circ} \mathrm{C}$ against $150 \mathrm{mM} \mathrm{NaCl}$. Liposomal DOX preparations were diluted with culture medium as necessary prior to cytotoxicity assay.

\section{Liposomal entrapment of PSC 833}

Standard solutions of PSC 833 were prepared in methanol and then evaporated to form a thin layer film. Following the addition of ELs or DOX-Ls, the mixtures were sonicated for $10 \mathrm{~min}$ at $60^{\circ} \mathrm{C}$, using a high-energy bath-type sonicator (Starsonic, Liarre, Italy). The liposomal suspensions were allowed to stand at room temperature for $30 \mathrm{~min}$. Unentrapped drug was separated by centrifugation at $13000 \mathrm{rpm}$ for 10 min.

\section{Liposome characterization}

The prepared liposomes were characterized in terms of entrapment efficiency (EE), vesicle size, zeta potential and in vitro drug release. 


\section{Entrapment efficiency}

Prepared lipid vesicles were separated from the free (unentrapped) drug. Encapsulated DOX in liposomal systems was determined spectrophotometrically at $480 \mathrm{~nm}$ after lysis of the liposomes with Triton X-100 (final concentration of $2 \% \mathrm{w} / \mathrm{v})$. For determination of PSC 833 entrapment efficiency, small aliquots of liposomes $(50 \mu \mathrm{l})$ were diluted in $950 \mu \mathrm{l}$ methanol, were subjected to sonication until liposomes disruption and analyzed for PSC 833 content by HPLC. The \%EE was calculated from the amount of incorporated drug divided by the total amount used at the beginning of preparation multiplied by 100 .

\section{PSC 833 analysis}

The amount of PSC 833 in liposome formulations and in release medium $(\mathrm{NaCl} 0.9 \%$ containing $0.05 \%$ Tween 80 ) was determined using a validated HPLC method. The HPLC system consisted of a model K-1001 solvent delivery pump, a Wellchrom online degasser, a Rheodyne autoinjector equipped with a $100 \mu \mathrm{l}$ loop, a model K-2600 UV detector (all from Knauer, Germany). Chromgate software (Version 317) was used to acquire and process all chromatographic data. The separation was achieved on a ShimPack CLC-CN column, $4.6 \times 250 \mathrm{~mm}, 5 \mu \mathrm{m}$ particle size (Shimadzu Co., Japan), using an isocratic mobile phase consisting of water and acetonitrile (40:60, $\mathrm{v} / \mathrm{v}$ ) as eluent at a flow rate of $1 \mathrm{ml} / \mathrm{min}$ with UV detection at $210 \mathrm{~nm}$. The column temperature was kept at $50^{\circ} \mathrm{C}$. A linear response was observed over a concentration range of $0.5-10.0 \mu \mathrm{g} / \mathrm{ml}$. The coefficients of variation for inter-day and intraday assay were found to be less than $6.0 \%$.

\section{Size distribution and zeta potential}

Size, population distribution and zeta potentials of EL, DOX-L, PSC-L and co-encapsulated liposomal formulation of DOX and PSC 833 (DOX/PSC-L) were determined by dynamic light scattering measurements using a Malvern Zetasizer Nano ZS (Malvern Instruments, London, England). The analysis was performed at $25^{\circ} \mathrm{C}$ and after the dispersion was diluted to an appropriate volume with deionized water. The measurements were conducted in triplicate. Particle size results are presented as an average diameter of the liposome suspension (z-average mean).

\section{In vitro drug release}

The in vitro release of drug loaded liposomal formulations was determined using home-made vertical Franz-type glass diffusion cells (available diffusion area $=1.8 \mathrm{~cm}^{2}$ ). A cellulose membrane (molecular weight cutoff of $12 \mathrm{KDa}$ ) was mounted between the donor and receptor compartments. The membrane was soaked in double-distilled water for 12 hours before mounting in the Franz diffusion cell. Respective liposomal formulations (DOX-L, PSC-L and DOX/PSC-L) were placed in donor compartment and dialyzed against receptor medium consisted of normal saline containing $0.05 \%$ Tween 80 (w/v) for achieving sink condition. The stirring rate and temperature were kept at $300 \mathrm{rpm}$ and $37^{\circ} \mathrm{C}$, respectively. The samples were removed at $1,2,4,6,8,24,48$ and 72 h. Each experiment was repeated in triplicate. In all cases the incubated medium was replaced with a fresh one daily. Samples for DOX analysis were taken from receptor and analyzed using a spectrofluorometric method at excitation and emission wavelengths of $485 \mathrm{~nm}$ and $580 \mathrm{~nm}$, respectively. With regard to PSC 833, samples were taken from donor and analyzed using above mentioned HPLC method.

\section{Stability of formulations}

The stability of liposomal formulations was evaluated at refrigerator temperature $\left(5^{\circ} \mathrm{C}\right)$ for a period of 15 days. At predetermined time intervals aliquots of samples were withdrawn and $\% \mathrm{EE}$, particle size and zeta potential of the liposomes were determined.

\section{In vitro cytotoxicity assay}

The in vitro cytotoxicity of various formulations against T47D/TAMR-6 cells was investigated by MTT assay. A $10^{4}$ T47D/TAMR-6 cells were cultured in 96-well plate containing RPMI medium and incubated overnight to allow cell attachment. After 48 hours incubation, fresh medium containing serial concentration of various drug formulations, including free DOX, DOX-L, mixture of DOX-L and free PSC 833, mixture of DOX-L and PSC-L and DOX/PSC-L were added. The plates were then incubated for an additional 48 hours before washing with normal saline followed by adding MTT solution $(0.5 \mathrm{mg} / \mathrm{ml})$ to each well, and incubated for $4 \mathrm{~h}$ at $37^{\circ} \mathrm{C}$. Then, the medium was removed, and DMSO was added to dissolve the formazan crystals. The plates were mildly shaken for $10 \mathrm{~min}$ to ensure the dissolution of formazan. The formazan dye was measured spectrophotometrically using microplate reader at $570 \mathrm{~nm}$ with reference standard of $690 \mathrm{~nm}$ as described before.

\section{STATISTICAL ANALYSIS}

All data were expressed as mean \pm standard deviation (SD) of at least three measurements. 
Statistical calculations were performed using GraphPad PRISM ${ }^{\mathrm{TM}}$ software. Statistical significance between two groups was tested with Student's t-test. Differences among three or more groups were analyzed by one-way analysis of variance (ANOVA) and Tukey's multiple comparison tests. A value of $\mathrm{P}<0.05$ was considered statistically significant.

\section{RESULTS}

\section{PSC 833 nanoliposome preparation}

There are many methods of liposome preparation and suitable method is selected according to the physical properties of the entrapped drugs. For PSC 833 liposome, the preparation was firstly attempted using the thin-film hydration technique, which is the conventional and most common technique for liposome preparation, followed by extrusion. However, a desired EE was not achieved. In the second step the ethanol injection method (31) was tried which was not effective due to the relatively large particle size of liposomes (around $210 \mathrm{~nm}$ ) and production of inhomogeneous population of particles; furthermore $\mathrm{EE}$ was rather low (EE $<20 \%$ for lipid composition of Chol:EPC 30:70 and lipid to drug molar ratio of 30). To achieve higher EE and minimizing the leakage of DOX during PSC 833 loading, we investigated the remote film loading method to prepare PSC 833 liposomes. This method was previously optimized in our lab for liposomalization of sirolimus (28).

\section{Entrapment efficiency}

In this study, in order to investigate the role of phospholipid composition on incorporation efficiency, different phospholipids, which vary in acyl chain length, the degree of saturation of the acyl chains and bilayer fluidity, were used for preparing different liposomal formulations. The chosen lipids are the most common used lipids for the liposome preparation. From Table 1, it can be readily noticed that $\mathrm{EE}$ closely correlated to the liposomal lipid composition. Immediately after formulation, EE was higher, however, when the sonicated liposomes were then allowed to stand undisturbed for about $30 \mathrm{~min}$, for annealing, a part of entrapped drug dissociated.

\begin{tabular}{|c|c|}
\hline Liposome formulation $^{\mathrm{a}}$ & $\% \mathrm{EE}$ \\
\hline Chol:DSPC30:70 & $10.1 \pm 1.3$ \\
\hline Chol:DPPC30:70 & $12.3 \pm 0.9$ \\
\hline Chol:EPC30:70 & $42.4 \pm 0.8$ \\
\hline Chol:EPC:DSPE-PEG2000 30:65:5 & $64.9 \pm 0.4$ \\
\hline${ }^{\mathrm{a}}$ Lipid to drug molar ratio $=30$ & \\
\hline
\end{tabular}

An increase in chain length of fatty acid resulted in a decrease in EE at a fixed lipid/drug ratio of 30 and Chol proportion of $30 \%$. Higher extent of incorporation was observed when using EPC liposomes (EE $\approx 42 \%$ ) compared to DPPCand DSPC-ones (EE $\approx 12$ and $10 \%$ respectively) (Table 1).

Considering all known desirable properties of PEG coated vesicles such as minimizing the recognition of injected liposomes by cells of the mononuclear phagocytic system, reducing liposome - liposome aggregation and improving long-term stability (21), a 5\% DSPE-PEG was incorporated in the EPC based formulations. Results revealed that inclusion of a small quantity of this PEGylated phospholipid had a distinctly positive influence on the EE (Table 1). Since lipid to drug molar ratio and Chol proportion could have significant impact on developing a therapeutically efficient liposomal carrier system, we examined the effect of these factors on different properties of the prepared liposomes. As shown in Table 2 the incorporated amount of PSC 833 displayed a lipid/drug molar ratio-dependent trend. In all cases, the amount of drug remaining associated with vesicles was always higher for greater lipid/drug ratios. The EE of liposomes markedly increased from approximately $22 \%$ to $65 \%$ by increasing lipid/drug ratio from 15 to 30 . Even though further increase of lipid/drug molar ratio from 30 to 45 favored the drug loading, we chose lipid/drug molar ratio of 30 for remaining studies because the amount of lipid that could be administered for a given drug dose is limited. High lipid doses may raise concerns of toxicity, reduce the economic feasibility of pharmaceutical scale production and worsen the physical characteristics of the dosage form. Increasing the content of Chol from $30 \%$ to $45 \%$ significantly affected the EE (Table 2). As the Chol content of the formulation increased, PSC 833 loading decreased.

\section{Particle size and zeta potential}

The mean particle size of liposome-incorporated PSC 833 with different lipid compositions, as measured with the particle size analyzer, was in the range of $90-110 \mathrm{~nm}$ with an acceptably good polydispersity allowing diffusion into malignant tissue via gaps present in the highly permeable tumor blood vessels. 
Table 2. Effects of lipid/drug molar ratio and cholesterol proportion on entrapment efficiency and release profile of PSC 833 loaded nanoliposomes (Mean $\pm \mathrm{SD}, \mathrm{n}=3$ ).

\begin{tabular}{|c|c|c|c|c|c|}
\hline \multicolumn{3}{|c|}{ Liposomal formulation } & \multirow{2}{*}{$\% \mathrm{EE}$} & \multirow{2}{*}{$\% \mathrm{DR}_{24 \mathrm{~h}^{\mathrm{a}}}$} & \multirow{2}{*}{$\% \mathrm{DR}_{48 \mathrm{~h}}^{\mathrm{b}}$} \\
\hline Main lipid & Lipid/drug ratio & $\%$ Chol & & & \\
\hline EPC & 15 & 30 & $21.8 \pm 0.2$ & $38.9 \pm 0.2$ & $43.5 \pm 1.1$ \\
\hline EPC & 30 & 30 & $64.9 \pm 0.4$ & $25.4 \pm 2.6$ & $37.6 \pm 6.8$ \\
\hline EPC & 45 & 30 & $73.3 \pm 0.5$ & $21.2 \pm 4.0$ & $27.6 \pm 5.0$ \\
\hline EPC & 30 & 45 & $30.4 \pm 0.6$ & $64.9 \pm 0.4$ & $66.1 \pm 0.8$ \\
\hline
\end{tabular}

Surface charge of nanoparticles is another important physiochemical parameters in designing drug delivery vehicles. Zeta potential is the electric charge on particle surface. Zeta potential of conventional empty vesicles and PSC-L were in the range of -3 to +1 . However, zeta potential of PEGylated vesicles found to be in the range of -10 to $-5 \mathrm{mv}$ due to the presence of the negatively charged DSPE-PEG. Aggregation of neutral liposomes is brought about by Van der Waals interactions. Small concentrations of PEGylated lipids can provide sufficient repulsion to prevent the aggregation of the particles upon addition of hydrophobic drugs to the membrane.

\section{In vitro drug release study}

The release profile of PSC 833 from liposomal formulations was studied in normal saline (containing $0.05 \% \mathrm{w} / \mathrm{v}$ Tween 80) (Table 2, Figure 1A). The release of PSC 833 from nanoliposomes was observed to occur as a biphasic process and the release rate of primary phase was faster than the steady release phase. From the results (Table 2), it can be noticed that Chol content and lipid/ drug molar ratio had a positive and a negative impact on the release rate, respectively.

\section{Doxorubicin nanoliposome preparation}

DOX is a cytotoxic agent which has been widely used in clinical cancer therapy. Ammonium sulfate gradient remote-loading method is robust to attain high encapsulation efficiency (more than $95 \%$, Table 3). The transmembrane ammonium sulphate gradient provided the driving force for DOX influx and accumulation within liposomes in an aggregated gel-like state (32).

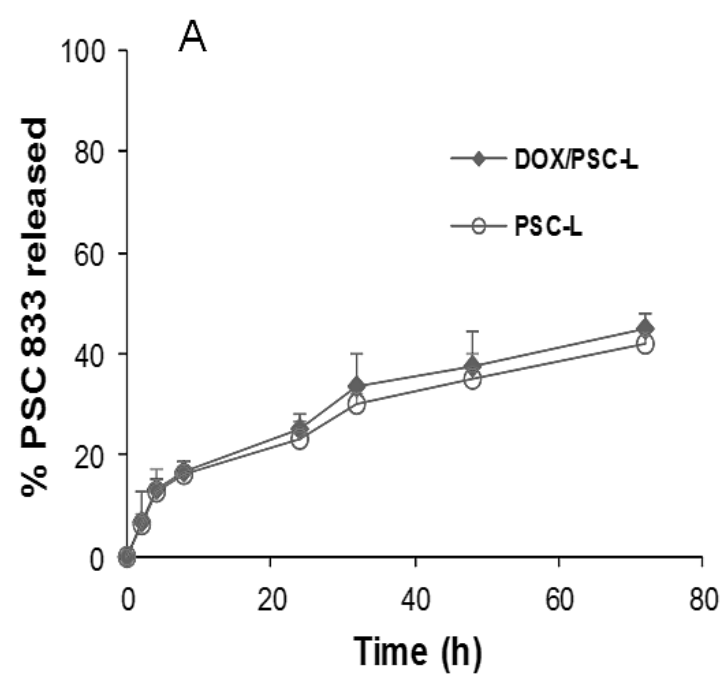

B

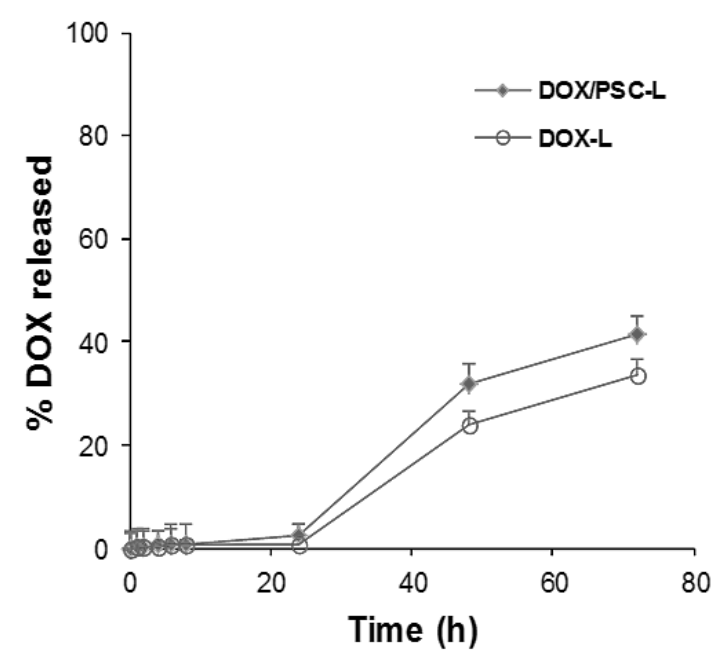

Figure 1. (A) In vitro release profile of PSC 833 from nanoliposomes with or without DOX. (B) In vitro release profile of DOX from nanoliposomes with or without PSC 833. Lipid composition was EPC/Chol/ DSPE-PEG2000 at a molar ratio of 65:30:5, (Mean \pm $\mathrm{SD}, \mathrm{n}=3$ ). 
The in vitro release studies of DOX-L (Figure 1B) showed minimal leakage and controlled release of DOX from liposomes in normal saline after 72 hours incubation at $37^{\circ} \mathrm{C}$. Within the interior of liposomes, rapid drug loss can be effectively prevented by the use of remote drug-loading techniques and formation of slow dissolving gellike aggregates. Liposomes were stable for at least 2 weeks at $5^{\circ} \mathrm{C}$ (Table 4).

\section{Formulation properties of dual agent stealth nanoliposomes}

The combined formulation (DOX/PSC-L) presented encapsulation contents close to those of each individual drug loaded formulation (Table 3 ). The presence of associated DOX did not alter the release profile of PSC 833 from the nanoparticles (Figure 1A). The presence of PSC 833 had no significant effect on the rate of DOX release (Figure 1B). Changing the formulation from single agent to dual agent, did not cause any significant change in the size or zeta potential of the particle (Table 3). Stability of DOX/PSC-L was also investigated (Table 4). In the present work, we have demonstrated that nanoliposomes can be formulated for efficient co-encapsulation and simultaneous controlled release of DOX and PSC 833, a hydrophilic and a lipophilic compound, respectively. This versatility property of liposomal system would be useful for the delivery of combinational treatments.

\section{Stability study of liposomes}

The results of the physical and chemical stability of various liposomal formulations at refrigerator temperature were shown in Table 4. The liposomes were stable for at least two weeks at this temperature without any detectable changes in EE, size and zeta potential.

\section{Cytotoxicity study}

The $\mathrm{IC}_{50}$ of DOX in T47D/TAMR-6 cells was significantly higher than that in T47D cells $(10$ $\mu \mathrm{g} / \mathrm{ml}$ and $1 \mu \mathrm{g} / \mathrm{ml}$ respectively, $\mathrm{p}<0.001)$, suggesting that T47D/TAMR-6 cells were resistant to effects of DOX. Cytotoxicities of various formulations of DOX were determined in resistant cells (T47D/TAMR-6) by an MTT assay. Empty liposomes (ELs) showed no toxicity in both wild type and MDR cells, reinforcing the biocompatibility of the liposomes. As illustrated in Figure 2, DOX-L had approximately 20 times lower IC50 value than that of free drug $(0.5 \mu \mathrm{g} / \mathrm{ml}$ and $10 \mu \mathrm{g} / \mathrm{ml}$ respectively) indicating that T47D/TAMR-6 cells were more sensitive to liposomal DOX than to free drug. These results confirm the advantage of small particle formulations of chemotherapeutic agents to enhance cellular drug uptake and retention (18).

\begin{tabular}{lcccc}
\hline \multicolumn{4}{l}{ Table 3. Loading efficiency, mean size and zeta potential of Doxorubicin and PSC 833 liposomes (Mean \pm SD, $\mathrm{n}=3$ ). } \\
\cline { 2 - 3 } Formulation $^{\mathrm{a}}$ & \multicolumn{2}{c}{ \%EE } & $\begin{array}{c}\text { Size } \\
(\mathrm{nm})\end{array}$ & Zeta potential \\
& PSC 833 & DOX & & \\
\cline { 2 - 3 } & - & & $90.2 \pm 0.7$ & $-7 \pm 0.9$ \\
DOX-L & $64.9 \pm 0.4$ & - & $93.6 \pm 0.3$ & $-5 \pm 1.4$ \\
PSC-L & $65.5 \pm 1.3$ & $>95$ & $91.3 \pm 0.2$ & $-6 \pm 1.2$ \\
DOX/PSC-L & \multicolumn{4}{c}{} \\
\hline
\end{tabular}

\begin{tabular}{lcccccccc}
\hline Table 4. Stability of various formulations at $5^{\circ} \mathrm{C}(\mathrm{Mean} \pm \mathrm{SD}, \mathrm{n}=3)$. \\
\hline Formulation $^{\mathrm{a}}$ & \multicolumn{2}{c}{ \%EE (DOX) } & \multicolumn{2}{c}{ \%EE (PSC 833) } & \multicolumn{2}{c}{ Size $(\mathrm{nm})$} & \multicolumn{2}{c}{ Zeta potential } \\
\cline { 2 - 9 } & Day 0 & Day 15 & Day 0 & Day 15 & Day 0 & Day 15 & Day 0 & Day 15 \\
\hline DOX-L & $98.3 \pm 0.5$ & $94.8 \pm 1.4$ & - & - & $90.2 \pm 0.7$ & $103 \pm 1.2$ & $-7 \pm 0.9$ & $-4 \pm 1.3$ \\
PSC-L & - & - & $64.9 \pm 0.4$ & $60.8 \pm 1.7$ & $93.6 \pm 0.3$ & $104 \pm 1.5$ & $-5 \pm 1.4$ & $-5 \pm 1.7$ \\
DOX/PSC-L & $97.9 \pm 0.4$ & $93.5 \pm 0.8$ & $65.9 \pm 1.3$ & $61.4 \pm 2.4$ & $91.3 \pm 0.2$ & $106 \pm 1.2$ & $-6 \pm 1.2$ & $-4 \pm 0.8$ \\
\hline
\end{tabular}


Prior to in vitro cytotoxicity work on coencapsulated formulations, the cytotoxicity of PSC 833 solutions at increasing concentration from $0.25 \mu \mathrm{g} / \mathrm{ml}$ to $0.75 \mu \mathrm{g} / \mathrm{ml}$ was also investigated. Consistent with previous reports that PSC can induce cell death at concentration of more than $10 \mu \mathrm{g} / \mathrm{ml}$ (33), the data of Figure 3 proved that no cytotoxicity effects were detected up to the concentration of $0.75 \mu \mathrm{g} / \mathrm{ml}$.
To determine the appropriate drug ratio that could give the best cytotoxic effect, DOX resistant cells were incubated with different concentrations of DOX-L plus free PSC 833 at concentrations of $0.25,0.5$ and $0.75 \mu \mathrm{g} / \mathrm{ml}$ (Figure 4). Cell kill efficacy of MDR cell type increased significantly when PSC 833 was administered alongside DOX-L.

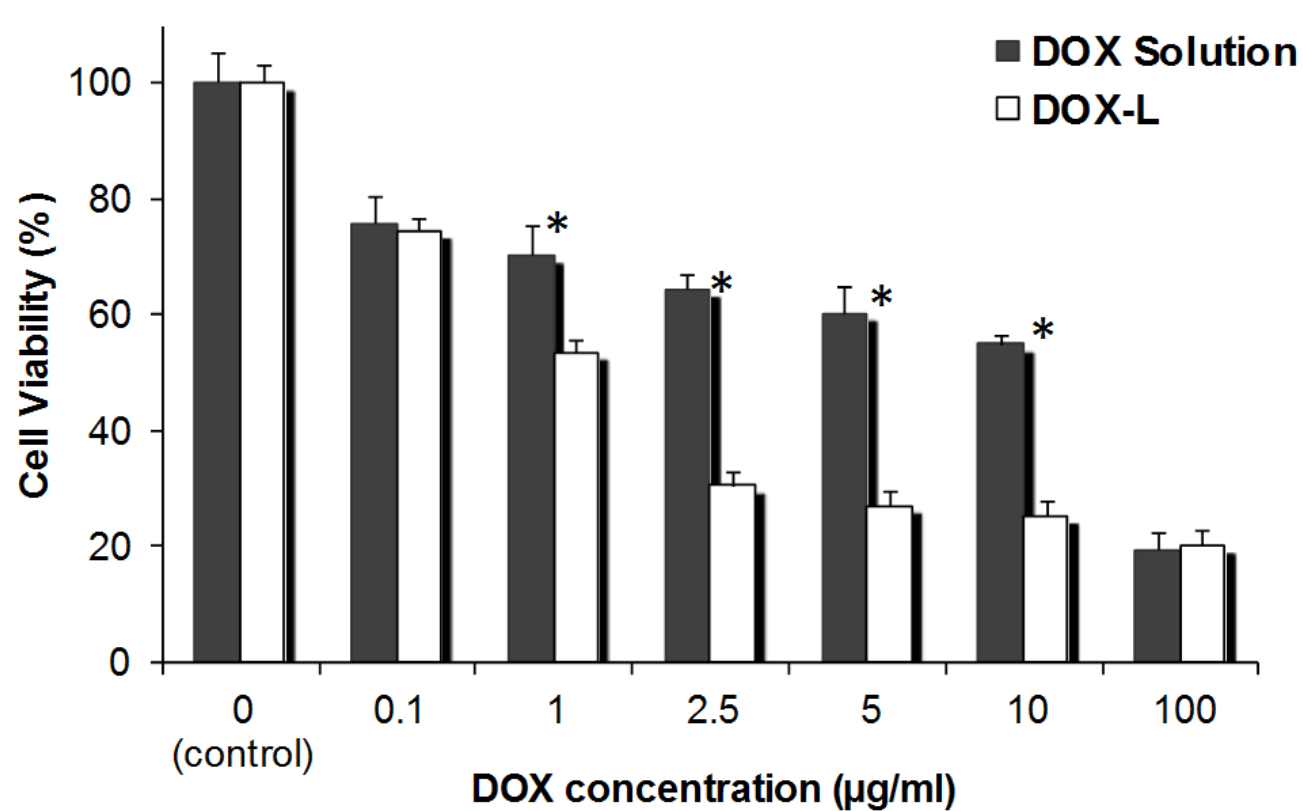

Figure 2. Cytotoxicity of free DOX and DOX loaded nanoliposomes against DOX resistant cells; $* \mathrm{P}<0.01$ (between free DOX and DOX-L)

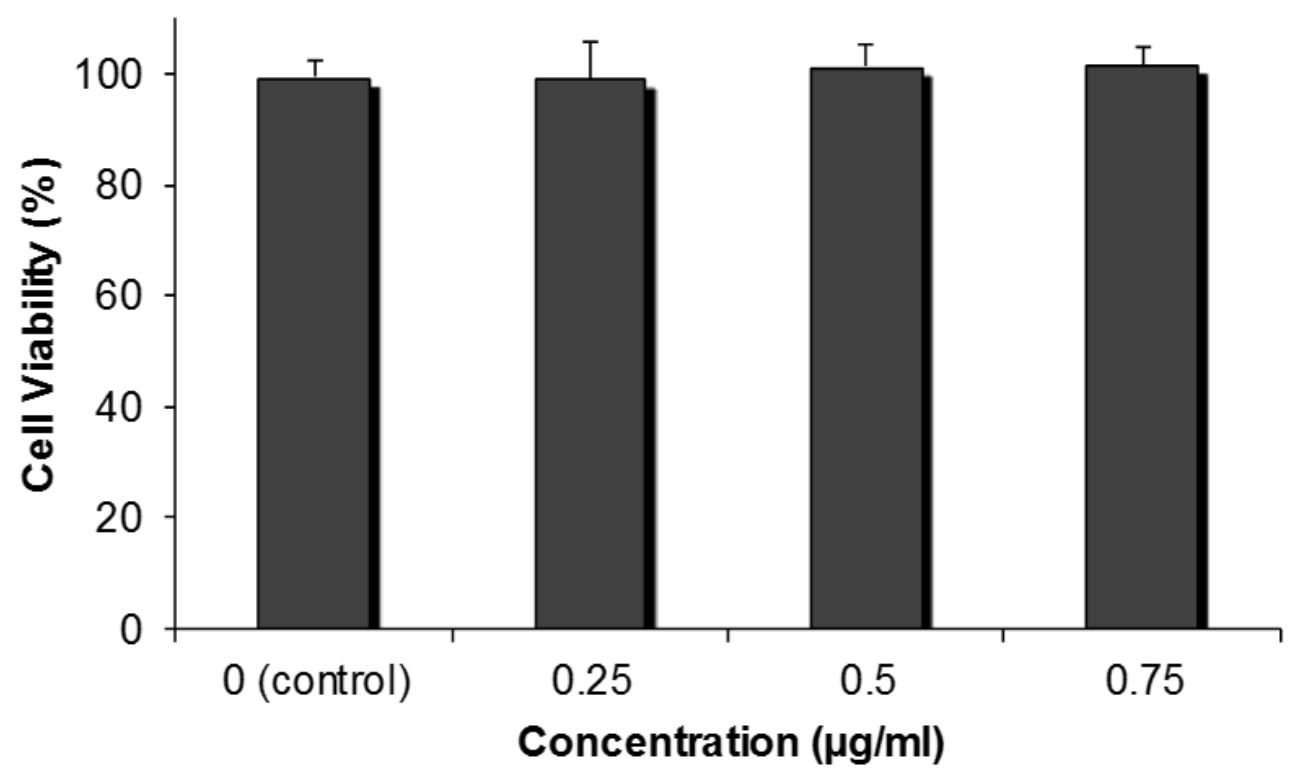

Figure 3. Cell viability after exposure to various concentrations of free PSC 833 , at $37^{\circ} \mathrm{C}$ for 48 hours. No significant differences were found in cell viability among different concentrations of PSC 833. 


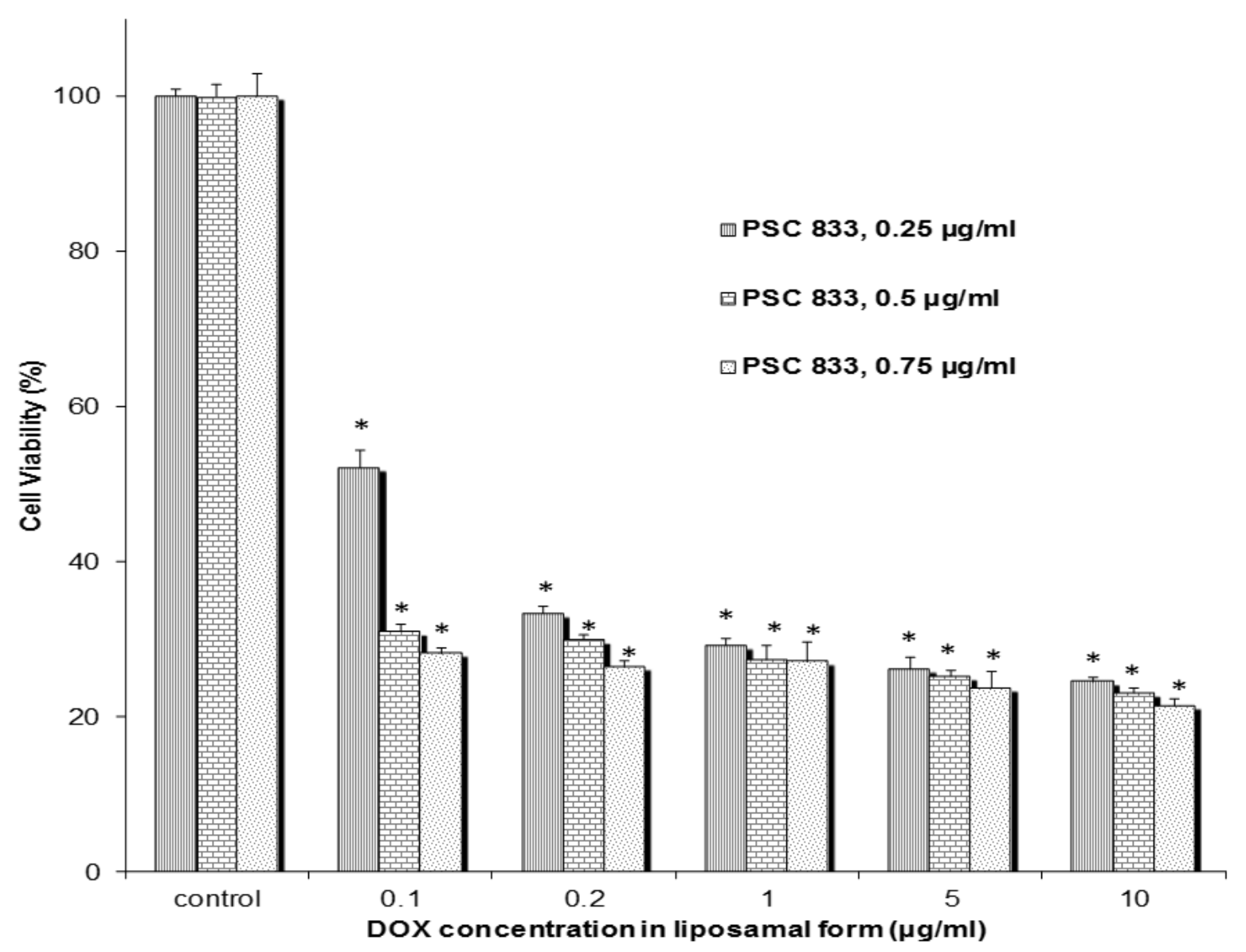

Figure 4. Cytotoxicity of different concentrations of DOX-loaded nanoliposomes (DOX-L) in the presence of various concentrations of free PSC $833 ; * \mathrm{P}<0.01$ (vs. the control).

According to the results, the higher concentrations of PSC $833(0.5$ and $0.75 \mu \mathrm{g} / \mathrm{ml})$, in combination with all concentrations of DOX, were most toxic and killed more than $70 \%$ of the resistant cells. However, for better discrimination and comparison of various formulations, a combination of $0.25 \mu \mathrm{g} / \mathrm{ml}$ of the chemosentisizer agent and $0.1 \mu \mathrm{g} / \mathrm{ml}$ of DOX were selected for further experiments. To incorporate this dose relationship $(0.1 \mu \mathrm{g} / \mathrm{ml}$ of DOX-L and $0.25 \mu \mathrm{g} / \mathrm{ml}$ of PSC 833) into the formulation, a stealth nanoliposomal system was designed that could simultaneously carry both the DOX and PSC 833 therapeutics but release each in a controlled manner (DOX/PSC-L).

Figure 5 compares the in vitro cell growth inhibition effect of various treatments containing DOX (with or without PSC 833) as measured by the MTT assay. As shown the efficiency of DOX against the resistant cells followed the order of DOX/PSC-L $\approx$ DOX-L + PSC-L > DOX-L + Free PSC $833>$ DOX-L > Free DOX. Clearly PSC 833-containing treatments were more effective and strong synergism was obtained in resistant cells. Although incorporation of PSC 833 within liposomal formulation led to an increase in efficacy in vitro $(\mathrm{P}<0.05)$ as compared to DOX$\mathrm{L}$ mixed with free PSC 833, the use of equal exposure time for free and liposomal formulation of PSC 833 in in vitro cytotoxicity studies creates an artificial condition. It could also be observed that the combination of the two separate nanoparticle formulations elicited the same in vitro effect than the co-encapsulated formulation (Figure 5).

\section{DISCUSSION}

Liposomes have been proven as successful nanocarriers because they prevent the drug from degradation in circulation and may diminish toxic side-effects by altering pharmacokinetic and distribution behavior. Furthermore, they can encapsulate or incorporate both hydrophilic and hydrophobic compounds in high quantities in their aqueous interior or in the lipid bilayer, 
respectively. Besides, it has been shown that liposomes may accumulate in tumor areas provided that they are small and have a sufficiently long circulation time $(22,34)$. Anthracyclines, which rank among the most widely employed chemotherapeutics, represent the main group of cytostatic drugs that have been associated with liposomes $(35,36)$. Liposomal formulations of doxorubicin and daunorubicin are either approved or under clinical investigation for treatment of several types of cancer, e.g. breast cancer, Kaposi's Sarcoma, ovarian cancer, nonHodgkin's lymphoma and multiple myeloma (3739).

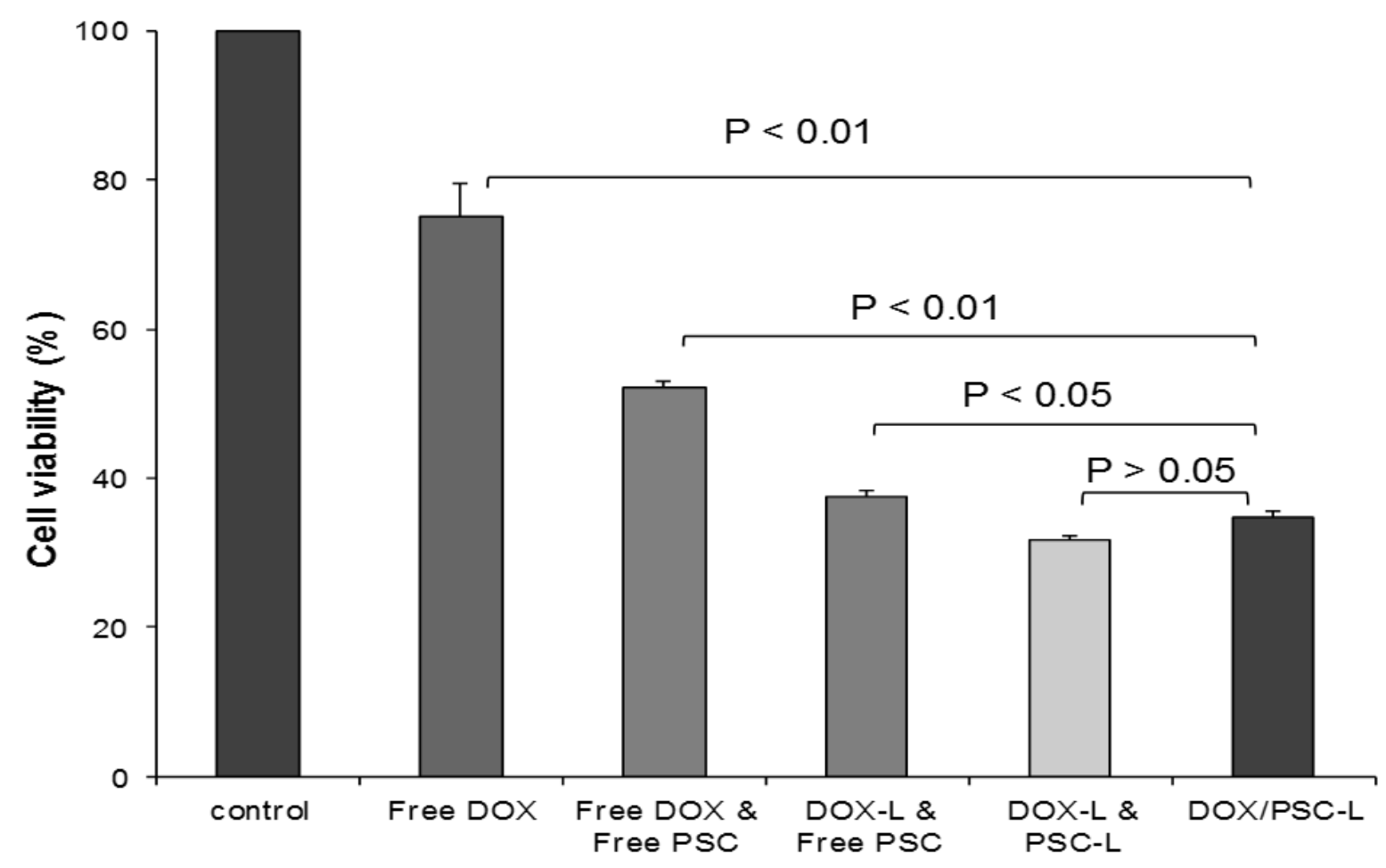

Figure 5. Cytotoxicity of various formulations of DOX against drug- resistant human breast cancer cell line, at $37^{\circ} \mathrm{C}$ for 48 hours. DOX: free doxorubicin; PSC: free PSC 833; DOX-L: doxorubicin loaded liposomes; PSC-L: PSC 833 loaded liposomes; DOX/PSC-L: co-encapsulated liposomal formulation of DOX and PSC 833.

Liposomal anthracyclin delivery has minimized non-specific biodistribution and reduced cardiotoxicity compared to free drug $(22,36)$. However, an important concern is that accumulation of liposomes in the tumor area does not guarantee intracellular bioavailability of the encapsulated drug. Intrinsic or acquired resistance to chemotherapeutic drugs is one of the major obstacles hampering effective cancer treatment. Functioning as a drug efflux pump, P-gp prevents cytotoxic agents from reaching effective intracellular concentrations (5). Simultaneous liposomal co-entrapment of chemosensitizers with anticancer drugs has been pursued as a means to increase chemotherapeutic efficacy, restore drug sensitivity while decreasing toxic effects. Liposomalization of specific P-gp inhibitors may provide therapeutic advantages by increasing their bioavailability at sites of tumor via the EPR effect and altered biodistribution. Considering all these issues, we aimed to develop DOX and PSC 833 co-loaded nanoliposomes that to our knowledge has not been prepared yet. The liposomes were evaluated for in vitro cytotoxicity in DOX resistant human breast cancer cell line.

For PSC 833 nanoliposome preparation, different methods were tried. A desired EE was not achieved with thin-film hydration technique followed by extrusion and the ethanol injection method. The remote loading methods using an ammonium sulphate or citrate gradient are another approach for liposome preparation which is successfully used for drugs that are weak bases like DOX by exerting a transmembrane $\mathrm{pH}$ gradient (40). For optimal loading, drug candidates are required to be amphiphilic weak bases (41) with $\log \mathrm{D}$ at $\mathrm{pH} 7$ in the range of -2.5 to 2 and a pKa $<11$ (42). However, drugs like PSC 833 that do not have the weakly basic amino groups, required for drug loading, cannot be stably encapsulated and retained in liposomal carriers by this method. On the other hand, the 
challenges in minimizing the leakage rate of PSC 833 during DOX encapsulation should be considered. Up to now, few studies have been reported on co-delivery of multiple agents in liposomes. This is probably due to difficulties associated with the efficient and stable encapsulation of two drugs with different physicochemical properties in the same liposome, as well as the challenges in controlling the release rate of chemically disparate drugs within one liposome composition (43). To overcome these problems, we investigated the remote film loading method to prepare PSC 833 liposomes. This method was reported by Sadzuka et al. (27) as a novel method for liposome preparation of SN-38 and optimized in our previous study for liposomalization of sirolimus (28).

First, the experiments were set up to investigate the effect of the different lipid compositions on the PSC 833-loaded vesicle formation. The selection of lipids for preparing liposomes to be used as a drug delivery system depends on many factors, including entrapment efficiency, as a key parameter in liposomal drug delivery, availability, cost, safety, and ease of utilization of the lipids. Encapsulation of a lipophilic drug depends, to a large degree, on the lipid composition $(44,45)$ and occurs mainly through partitioning into the membrane (46). For DPPC and DSPC increase in the fatty acid chain length and the gel state of liposomes composed of these lipids are the factors responsible for reduced liposomal entrapment of PSC 833. In the presence of rigid acyl chain of DPPC and DSPC freedom of movement of highly lipophilic and bulky molecules of PSC 833 may decrease. However, EPC is a mixture of saturated and unsaturated acyl chains with a $\mathrm{Tm}$ ranging from -2.5 to $-15{ }^{\circ} \mathrm{C}$ (34). In EPC liposome, phospholipids are in the liquid crystalline state and regions of high bilayer disorder exist. Generally hydrophobic drugs tend to be incorporated more efficiently in fluid membranes where the phospholipid acyl side chains and the drug molecules have considerable freedom of movement. From the present investigation, the relatively higher loading efficiency of PSC 833 in EPC liposomes suggests that a fluid lipid mixture would be a better choice for PSC 833 entrapment. In contrast, higher encapsulation of hydrophilic compounds into the DPPC- and DSPC-liposomes compared to more fluid liposomes which were shown by (47) might be explained by the higher rigidity of the former, which minimize the leakage of entrapped materials.

Although appropriately designed conventional liposomes prepared of phospholipids and Chol could improve drug delivery to diseased sites and organs, it was proposed that additional benefits would be achieved if these conventional formulations were superseded by second generation liposomes termed "sterically stabilized" or Stealth, that their surface was coated with synthetic neutral polymer polyethylene glycol (PEG) (21). PEG coating exhibits decreased rates of removal by the mononuclear phagocyte system, consequently, prolong liposomal drug circulation time, reduce liposome - liposome aggregation and improve long-term stability of liposomes $(21,48)$. It has also been demonstrated that PEG liposomes can reach tumor tissue via high and leaky vasculature of tumor (49). Furthermore, incorporation of PEG modified lipids has provided flexibility in altering the liposome composition.

As shown in Table 2, the amount of drug remaining associated with vesicles is always higher for lower drug/lipid ratios. This may be due to two reasons. First, increasing the lipid concentration level certainly leads to an increase in the number of liposomes present per milliliter and hence there is more drug per milliliter of the formulation (50). Second, passive bilayer partitioning of lipophilic PSC 833 would also increase with increasing lipid concentration and number of liposomes. This observation is in agreement with the results of Nallamothu et al. (46) and Xiong et al. (51).

By changing the Chol content in liposomal formulations containing PSC 833, we were able to identify that the incorporation of PSC 833 was decreased as Chol concentration in the membranes increased. Chol is known to increase membrane rigidity and packing density by accumulating in the molecular cavities formed by the phospholipid molecules assembled into bilayer vesicles (52) which may result in decreased bilayer partitioning and hydrophobic space available for incorporation of hydrophobic drugs like PSC 833. This phenomenon was also reported for other lipophilic drugs such as paclitaxel (53) and nystatin (54). Based on the obtained results a lipid composition of EPC/Chol/ DSPE-PEG2000 at a molar ratio of 65:30:5 was chosen for preparation of single agent and coencapsulated liposomes (DOX-L, PSC-L and DOX/PSC-L).

For a formulation to be effective, it must provide high drug loading capabilities, as well as the ability to control release of drug cargo. Nanoliposomes provide a platform for the sustained release of drugs (22). According to the results of in vitro release studies, the release of PSC 833 from liposomes was adequately slow 
and occurred as a biphasic process. Considering that PSC 833 is mainly associated within the bilayer lipid structure of the liposomes, the initial release is primarily ascribed to drug detachment from outer lamellae and drug adsorbed on or close to the surface of the particles. Furthermore, smaller particle of nanoliposomes is associated with smaller diffusion path, so drug accessible to dissolution medium interface can diffuse easily. The later slow release results from sustained drug release from the inner lamellae and is assumed to be controlled by diffusion rate of drug across the liposomal bilayer. Incorporation of higher $\mathrm{Chol}$ concentration in liposomal formulations increased the PSC 833 release rate. The planar and rigid ring system of Chol is thought to reside in outer layer part of the fatty acyl chain region where it tends to restrict the motion of chains in liquid crystalline bilayers $(55,56)$ which may increase tendency of PSC 833 to partition in the release medium. We have previously reported phospholipids concentration-dependent drug release from liposomes (28). A simple explanation for this observation is that as the concentration of lipids increases, the number of liposomes in the dispersion is increased proportionally, however the number of PSC 833 molecules in bilayer of each liposome decreased correspondingly. As a result cohesive and hydrophobic interaction between hydrocarbon chains of phospholipids and the remaining PSC 833 molecules retarded drug release more efficiently. These findings suggest that, the release behavior can be controlled by tuning the lipid concentration.

Even though stealth liposome encapsulating DOX (DOX-L) improved drug delivery to resistant tumor cells, DOX-L alone was not enough to reverse the resistance of this cell line. In fact adding PSC 833 to the DOX-L led to an increased effectiveness which can be attributed to the P-gp modulation by the PSC 833 (12) and therefore diminished DOX efflux from the cells. The dual- agent loaded liposomal formulation as well as the combination of single-drug loaded liposomes was the most effective and the free DOX was the least effective treatments. The cytotoxicity demonstrated by the mixture of DOX-L and PSC-L and by the mixture of DOX-L and free PSC 833 fall in the middle of the spectrum.

Although DOX-L plus PSC-L showed the same degree of cell killing as DOX/PSC-L, simultaneous delivery of a particle formulation containing both drugs allows ease of administration. Moreover, co-loading DOX and PSC 833 ensures that following an in-vivo administration, a high local concentration of DOX and PSC 833 overlap with each other. Consequently, the P-gp transporters that are exposed to high DOX concentration will be strongly inhibited by the PSC 833 molecules that are in the close proximity. As a result any cellular uptake of liposomes would result in the exploitation of the synergy and cell killing.

Furthermore, co-encapsulation of both drugs in a single formulation reduces the amount of the lipids as compared to the amount required in two separate nanoparticle dosage forms, allowing a better safety profile of the combined formulation.

The enhanced antitumor efficacy of the coencapsulated stealth nanoliposomes, here noted in vitro, is expected to be valid for the in vivo situation as well. However, in vitro drug-release kinetics may not reflect drug-release kinetics under tumor mass conditions. This remains an important area for further investigation.

\section{Limitations}

Some limitations of the present study need to be acknowledged. The efficacy of this novel drug delivery system in other multidrug resistant cell lines which overexpress P-glycoprotein remain to be evaluated. Moreover, the in vivo efficacy parameters such as tumor volume and weight, tumor morphology assessment and immunohistochemistry of tumor sections are required to be measured in appropriate animal model. Finally, pharmacokinetic and tissue distribution studies in animal model of MDR human breast cancer could provide helpful data for future investigations

\section{CONCLUSION}

In the current study, co-encapsulated DOX and PSC 833 liposomes were prepared and characterized regarding their size, loading efficiency and in vitro drug release. The potential of dual-agent incorporated nanoliposomes to overcome P-gp mediated MDR was also investigated. Cell kill efficacy of MDR cell type increased significantly when PSC 833 was administered alongside DOX in liposomal formulation. Therefore, co-encapsulated DOX and PSC 833 liposomes can be proposed to have potential as a therapeutic approach to overcome the chemoresistance. In other words, by integrating two drug-resistance reversal strategies, namely, the use of a nanoparticulate drug delivery system along with chemosensitization, additional therapeutic benefit can be obtained. 


\section{ACKNOWLEDGMENTS}

The authors acknowledge Prof. Azizi (Tehran University of Medical Sciences) for providing DOX resistant cells; Ms Zahra Abbasian, Ms Leila Astaraki for their valuable technical assistance and Novartis Pharmaceuticals (Switzerland) for providing PSC 833. This work was supported in part by a grant from Shahid Beheshti University of Medical Sciences.

\section{CONFLICT OF INTEREST}

The authors report no conflicts of interest.

\section{REFERENCES}

1. Gottesman MM, Fojo T, Bates SE. Multidrug resistance in cancer: role of ATP-dependent transporters. Nat Rev Cancer, 2002; 2: 48-58.

2. Reddy LH. Drug delivery to tumours: recent strategies. J Pharm Pharmacol, 2005; 57: 12311242.

3. Stavrovskaya AA. Cellular mechanisms of multidrug resistance of tumor cells. Biochemistry (Mosc), 2000; 65: 95-106.

4. Vaalburg W, Hendrikse NH, Elsinga PH, Bart J, van Waarde A. P-glycoprotein activity and biological response. Toxicol Appl Pharmacol, 2005; 207: 257-260.

5. Goda K, Bacso Z, Szabo G. Multidrug resistance through the spectacle of P-glycoprotein. Curr Cancer Drug Targets, 2009; 9: 281-297.

6. Trock BJ, Leonessa F, Clarke R. Multidrug resistance in breast cancer: a meta-analysis of MDR1/gp170 expression and its possible functional significance. J Natl Cancer Inst, 1997; 89: 917-931.

7. Chintamani, Singh JP, Mittal MK, et al. Role of pglycoprotein expression in predicting response to neoadjuvant chemotherapy in breast cancer--a prospective clinical study. World J Surg Oncol, 2005; 3: 61.

8. Ford JM, Hait WN. Pharmacologic circumvention of multidrug resistance. Cytotechnology, 1993; 12: 171-212.

9. Krishna R, Mayer LD. Multidrug resistance (MDR) in cancer. Mechanisms, reversal using modulators of MDR and the role of MDR modulators in influencing the pharmacokinetics of anticancer drugs. Eur J Pharm Sci, 2000; 11: 265283.

10. Varma MV, Ashokraj Y, Dey CS, Panchagnula R. P-glycoprotein inhibitors and their screening: a perspective from bioavailability enhancement. Pharmacol Res, 2003; 48: 347-359.

11. Boesch D, Muller K, Pourtier-Manzanedo A, Loor F. Restoration of daunomycin retention in multidrug-resistant P388 cells by submicromolar concentrations of SDZ PSC 833, a nonimmunosuppressive cyclosporin derivative. Exp Cell Res, 1991; 196: 26-32.

12. Loor F. Valspodar: current status and perspectives. Expert Opin Investig Drugs, 1999; 8: 807-835.

13. Chico I, Kang MH, Bergan R, et al. Phase I study of infusional paclitaxel in combination with the $\mathrm{P}$ glycoprotein antagonist PSC 833. J Clin Oncol, 2001; 19: 832-842.

14. Bates SE, Bakke S, Kang M, et al. A phase I/II study of infusional vinblastine with the $\mathrm{P}$ glycoprotein antagonist valspodar (PSC 833) in renal cell carcinoma. Clin Cancer Res, 2004; 10: 4724-4733.

15. Advani R, Saba HI, Tallman MS, et al. Treatment of refractory and relapsed acute myelogenous leukemia with combination chemotherapy plus the multidrug resistance modulator PSC 833 (Valspodar). Blood, 1999; 93: 787-795.

16. McLeod HL. Clinical reversal of the multidrug resistance phenotype: true tumour modulation or pharmacokinetic interaction? Eur J Cancer, 1994; 30A: 2039-2041.

17. Shuhendler AJ, Cheung RY, Manias J, Connor A, Rauth AM, Wu XY. A novel doxorubicinmitomycin $\mathrm{C}$ co-encapsulated nanoparticle formulation exhibits anti-cancer synergy in multidrug resistant human breast cancer cells. Breast Cancer Res Treat, 2010; 119: 255-269.

18. Chidambaram M, Manavalan R, Kathiresan K. Nanotherapeutics to overcome conventional cancer chemotherapy limitations. J Pharm Pharm Sci, 2011; 14: 67-77.

19. Yuan F, Leunig M, Huang SK, Berk DA, Papahadjopoulos D, Jain RK. Microvascular permeability and interstitial penetration of sterically stabilized (stealth) liposomes in a human tumor xenograft. Cancer Res, 1994; 54: 33523356.

20. Gabizon AA. Stealth liposomes and tumor targeting: one step further in the quest for the magic bullet. Clin Cancer Res, 2001; 7: 223-225.

21. Immordino ML, Dosio F, Cattel L. Stealth liposomes: review of the basic science, rationale, and clinical applications, existing and potential. Int J Nanomedicine, 2006; 1: 297-315.

22. Drummond DC, Noble CO, Hayes ME, Park JW, Kirpotin DB. Pharmacokinetics and in vivo drug release rates in liposomal nanocarrier development. J Pharm Sci, 2008; 97: 4696-4740.

23. Wang J, Goh B, Lu W, et al. In vitro cytotoxicity of Stealth liposomes co-encapsulating doxorubicin and verapamil on doxorubicin-resistant tumor cells. Biol Pharm Bull, 2005; 28: 822-828.

24. Wang JC, Liu XY, Lu WL, et al. Pharmacokinetics of intravenously administered stealth liposomal doxorubicin modulated with verapamil in rats. Eur J Pharm Biopharm, 2006; 62: 44-51.

25. Motahari Z, Etebary M, Azizi E. Studying the role of p-glycoprotein in resistance to tamoxifen in human breast cancer T47D cells by immunocytochemistry. Int J Pharmacol, 2005; 1: 12-17. 
26. Charrois GJ, Allen TM. Drug release rate influences the pharmacokinetics, biodistribution, therapeutic activity, and toxicity of pegylated liposomal doxorubicin formulations in murine breast cancer. Biochim Biophys Acta, 2004; 1663: 167-177.

27. Sadzuka Y, Takabe H, Sonobe T. Liposomalization of SN-38 as active metabolite of CPT-11. J Control Release, 2005; 108: 453-459.

28. Haeri A, Sadeghian S, Rabbani S, Anvari MS, Boroumand MA, Dadashzadeh S. Use of remote film loading methodology to entrap sirolimus into liposomes: Preparation, characterization and in vivo efficacy for treatment of restenosis. Int $\mathrm{J}$ Pharm, 2011; 414: 16-27.

29. Hope MJ, Bally MB, Webb G, Cullis PR. Production of large unilamellar vesicles by a rapid extrusion procedure. Characterization of size distribution, trapped volume and ability to maintain a membrane potential. Biochimica et Biophysica Acta (BBA) - Biomembranes, 1985; 812: 55-65.

30. Stewart JC. Colorimetric determination of phospholipids with ammonium ferrothiocyanate. Anal Biochem, 1980; 104: 10-14.

31. Meure LA, Foster NR, Dehghani F. Conventional and dense gas techniques for the production of liposomes: a review. AAPS PharmSciTech, 2008; 9: 798-809.

32. Haran G, Cohen R, Bar LK, Barenholz Y. Transmembrane ammonium sulfate gradients in liposomes produce efficient and stable entrapment of amphipathic weak bases. Biochim Biophys Acta, 1993; 1151: 201-215.

33. Sadanand V, Kankesan J, Yusuf A, et al. Effect of PSC 833, a potent inhibitor of P-glycoprotein, on the growth of astrocytoma cells in vitro. Cancer Lett, 2003; 198: 21-27.

34. Drummond DC, Meyer O, Hong K, Kirpotin DB, Papahadjopoulos D. Optimizing liposomes for delivery of chemotherapeutic agents to solid tumors. Pharmacol Rev, 1999; 51: 691-743.

35. Minotti G, Menna P, Salvatorelli E, Cairo G, Gianni L. Anthracyclines: molecular advances and pharmacologic developments in antitumor activity and cardiotoxicity. Pharmacol Rev, 2004; 56: 185229.

36. Patil RR, Guhagarkar SA, Devarajan PV. Engineered nanocarriers of doxorubicin: a current update. Crit Rev Ther Drug Carrier Syst, 2008; 25 : $1-61$.

37. Alberts DS, Muggia FM, Carmichael J, et al. Efficacy and safety of liposomal anthracyclines in phase I/II clinical trials. Semin Oncol, 2004; 31: 53-90.

38. Krown SE, Northfelt DW, Osoba D, Stewart JS. Use of liposomal anthracyclines in Kaposi's sarcoma. Semin Oncol, 2004; 31: 36-52.

39. Robert NJ, Vogel CL, Henderson IC, et al. The role of the liposomal anthracyclines and other systemic therapies in the management of advanced breast cancer. Semin Oncol, 2004; 31: 106-146.
40. Webb MS, Johnstone S, Morris TJ, et al. In vitro and in vivo characterization of a combination chemotherapy formulation consisting of vinorelbine and phosphatidylserine. Eur J Pharm Biopharm, 2007; 65: 289-299.

41. Harrigan PR, Wong KF, Redelmeier TE, Wheeler JJ, Cullis PR. Accumulation of doxorubicin and other lipophilic amines into large unilamellar vesicles in response to transmembrane $\mathrm{pH}$ gradients. Biochim Biophys Acta, 1993; 1149: 329-338.

42. Zucker D, Marcus D, Barenholz Y, Goldblum A. Liposome drugs' loading efficiency: a working model based on loading conditions and drug's physicochemical properties. J Control Release, 2009; 139: 73-80.

43. Tardi PG, Gallagher RC, Johnstone S, et al. Coencapsulation of irinotecan and floxuridine into low cholesterol-containing liposomes that coordinate drug release in vivo. Biochim Biophys Acta, 2007; 1768: 678-687.

44. Betageri GV, Parsons DL. Drug encapsulation and release from multilamellar and unilamellar liposomes. Int J Pharm, 1992; 81: 235-241.

45. Haeri A, Sadeghian S, Rabbani S, Anvari MS, Erfan M, Dadashzadeh S. PEGylated estradiol benzoate liposomes as a potential local vascular delivery system for treatment of restenosis. J Microencapsul, 2012; 29: 83-94.

46. Nallamothu R, Wood GC, Kiani MF, Moore BM, Horton FP, Thoma LA. A targeted liposome delivery system for combretastatin A4: formulation optimization through drug loading and in vitro release studies. PDA J Pharm Sci Technol, 2006; 60: 144-155.

47. Vali AM, Toliyat T, Shafaghi B, Dadashzadeh S. Preparation, optimization, and characterization of topotecan loaded PEGylated liposomes using factorial design. Drug Dev Ind Pharm, 2008; 34: $10-23$.

48. Allen TM, Hansen C. Pharmacokinetics of stealth versus conventional liposomes: effect of dose. Biochim Biophys Acta, 1991; 1068: 133-141.

49. Harrington KJ, Mohammadtaghi S, Uster PS, et al. Effective targeting of solid tumors in patients with locally advanced cancers by radiolabeled pegylated liposomes. Clin Cancer Res, 2001; 7: 243-254.

50. Zuidam, JZ., de Vrueh, R., Crommelin, DJA., Characterization of Liposomes. In: Torchilin VP, Weissig V. (eds.), Liposomes, a Practical Approach. Oxford University Press, New York, USA, pp 31-78, 2003.

51. Xiong Y, Guo D, Wang L, Zheng X, Zhang Y, Chen J. Development of nobiliside A loaded liposomal formulation using response surface methodology. Int J Pharm, 2009; 371: 197-203.

52. Semple SC, Chonn A, Cullis PR. Influence of cholesterol on the association of plasma proteins with liposomes. Biochemistry, 1996; 35: 25212525.

53. Zhang JA, Anyarambhatla G, Ma L, et al. Development and characterization of a novel 
Cremophor EL free liposome-based paclitaxel (LEP-ETU) formulation. Eur J Pharm Biopharm, 2005; 59: 177-187.

54. Moribe K, Maruyama K, Iwatsuru M. Encapsulation characteristics of nystatin in liposomes: effects of cholesterol and polyethylene glycol derivatives. Int J Pharm, 1999; 188: 193202.

55. Robinson AJ, Richards WG, Thomas PJ, Hann MM. Behavior of cholesterol and its effect on head group and chain conformations in lipid bilayers: a molecular dynamics study. Biophys J, 1995; 68: 164-170.

56. Lagerquist C, Beigi F, Karlen A, Lennernas H, Lundahl P. Effects of cholesterol and model transmembrane proteins on drug partitioning into lipid bilayers as analysed by immobilizedliposome chromatography. J Pharm Pharmacol, 2001; 53: 1477-1487. 